\title{
Adequate cage placement for a satisfactory outcome after lumbar lateral interbody fusion with MRI and CT analysis
}

\author{
Shigeto Ebata, Tetsuro Ohba and Hirotaka Haro \\ Department of Orthopaedic Surgery, Graduate School of Medicine, University of Yamanashi, Yamanashi, Japan
}

\begin{abstract}
:
Introduction: Through an extreme lateral retroperitoneal and transpsoas approach to intervertebral disc and fusion surgery, a large lordosis cage can be placed for solid and stable intervertebral fusion and to provide strong anterior support, disc height restoration, favorable alignment, and indirect nerve decompression. However, appropriate placement of the interbody cage remains insufficiently researched. We sought to determine both appropriate cage placement as well as other factors affecting nerve decompression in extreme lateral interbody fusion (XLIF) surgery.
\end{abstract}

Methods: We included 53 consecutive patients suffering from lumbar degenerative diseases with an indication for XLIF. Radiographic analysis using a sagittal computed tomography (CT) and axial magnetic resonance imaging (MRI) views was conducted to determine intervertebral disc height and angle, degree of disc bulging and thickness of the flavum, the area of the dural tube, cage height, pre- and postoperative disc bulging, change of disc bulging after surgery, cage subsidence, and cage placement at the rostral and caudal endplates.

Results: Intervertebral disc height and angle were significantly increased at all levels $(\mathrm{L} 2 / 3,3 / 4,4 / 5)(p<0.05)$. The area of the dural tube was significantly increased $(\mathrm{p}<0.05)$, whereas the degree of disc bulging and thickness of the flavum were significantly decreased at all disc levels $(\mathrm{p}<0.05)$. The enlarged area of the dural tube showed significant correlation with increased disc height $(p=0.019)$, preoperative flavum thickness $(p=0.008)$, change of flavum thickness $(p<0.0001)$, and cage placement at the rostral endplate $(\mathrm{p}=0.014)$.

Conclusions: A decrease in flavum buckling is more important than disc protrusion as a consideration for obtaining indirect decompression. Central placement may be advantageous for indirect decompression.

Keywords:

XLIF, indirect decompression, flavum buckling, cage placement

Spine Surg Relat Res 2018; 2(1): 53-59 dx.doi.org/10.22603/ssrr.2017-0037

\section{Introduction}

Normal aging changes in the lumbar spine result in spinal canal stenosis with intracanal bony proliferation, buckling of the hypertrophic ligamentum flavum, and loss of disc height through degeneration, leading to the compression of the nerve roots or cauda equina ${ }^{1)}$. Therefore, surgical treatment for lumbar spinal stenosis should involve complete decompression of the narrow segment by partial resection of the lamina and central part of the facet process and the removal of the flavum to confirm decompression of the dural tube in the central canal and the nerve root in the lateral recess ${ }^{2,3)}$. Patients with stenosis in the region of the foraminal zone, abnormal segmental mobility derived from spondylolisthesis, and adult spine deformity are required to have not only decompression but also correction and fusion surgery with instrumentation $^{3)}$. An advantage of decompression surgery is encountering and recognizing compressed nerve tissue in the stenotic region under direct vision; whereas various adverse effects include hematoma, dural tear and liquorrhea, iatrogenic intervertebral instability, infection, and massive postoperative bleeding ${ }^{3,4)}$. Complications such as deep venous thrombosis and intraoperative damage to ureters and sympathetic nerves have been reported in cases of anterior lumbar interbody fusion ${ }^{5-7)}$. In addition, a surgical plan for patients with adult spinal deformity is required to obtain adequate global spinal balance.

A lateral retroperitoneal and transpsoas approach to the

Corresponding author: Hirotaka Haro, haro@yamanashi.ac.jp

Received: May 1, 2017, Accepted: July 4, 2017

Copyright (C) 2018 The Japanese Society for Spine Surgery and Related Research 
intervertebral disc is safe, causes less bleeding, and more quickly reaches the target region compared with traditional anterior transperitoneal or extraperitoneal approaches ${ }^{8,9)}$. Neuroelectromyographic monitoring is required to avoid damages to the nerve plexus. After dissecting and thorough disc removal, we place a disc cage relatively larger, longer, and wider than the cages used for posterior lateral interbody fusion. This new surgical procedure is called extreme lateral interbody fusion (XLIF; Nu Vasive, San Diego, CA). A large lordosis cage for XLIF is required for solid and stable intervertebral fusion, which provides a strong anterior support for interbody distraction and disc height restoration and for favorable sagittal and coronal physiological curvature ${ }^{10,11}$. In addition, the current procedure results in correction of spondylolisthesis and rotatory deformity, indirect nerve decompression by ligamentotaxis force, and a favorable bony union $^{12}$. However, the appropriate interbody cage placement after discectomy has not been fully elucidated. In addition, end plate damage when a cage is placed or sequential cage subsidence into an end plate may result in incomplete disc height and nerve decompression. Therefore, the purpose of the present study was to determine an appropriate cage placement to obtain effective indirect decompression and to determine factors for nerve decompression in the XLIF procedure.

\section{Patients and methods}

\section{Patients and study measures}

This prospective cohort study was approved by our institutional review board. All patients included had not responded to nonsurgical treatments with medication and/or orthosis for at least 6 months after first beginning these treatments and were therefore indicated for surgery to treat lumbar spinal stenosis associated with neurological deterioration. All patients underwent surgery by a single boardcertified surgeon. Patients with previous back surgery were excluded.

Radiography was conducted using sagittal computed tomography (CT)-multiplanar reconstruction (MPR) and axial T2-weighted magnetic resonance imaging (MRI) to determine intervertebral disc height and angle, cage height, degree of disc bulging, and thickness of the flavum before and after surgery, the area of the dural tube, and postoperative enlarged area of dural tube (Synapse, Fuji Film Medical, Tokyo, Japan). Intervertebral disc height was defined as the average of anterior and posterior height from CT-MPR in a sagittal view. The degree of intervertebral disc bulging was expressed as the distance between the tip of the bulging disc and a line connected with the posterior edge of rostral and caudal vertebral bodies. To determine placement of the cage, we measured the distance (a) between the anterior edge and the center of the cage on both the rostral and caudal end-

A
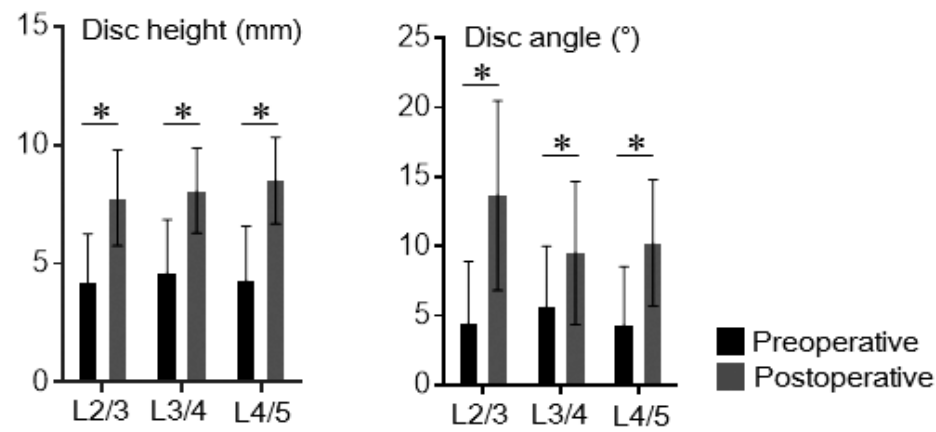

B
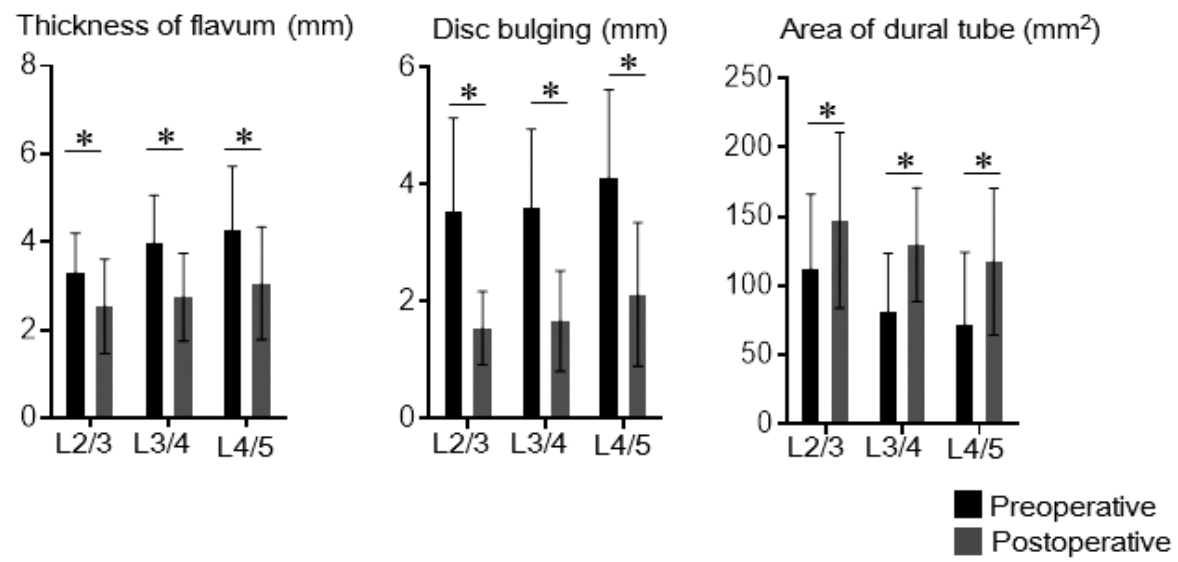

Figure 1. Measurements from a sagittal view of CT-MPR. (A) Disc height (mm) and angle $\left(^{\circ}\right)$ was examined before (black scale) and after (gray scale) surgery. Statistical analysis was performed using an unpaired $t$ test. Significant changes after surgery were at a level of $* p<0.05$. 
A

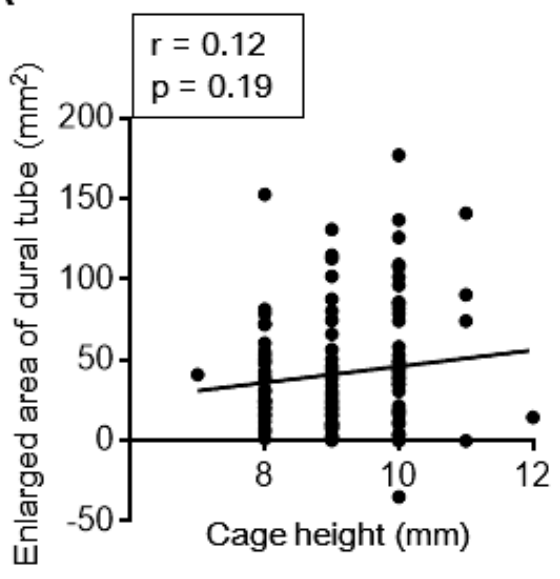

C

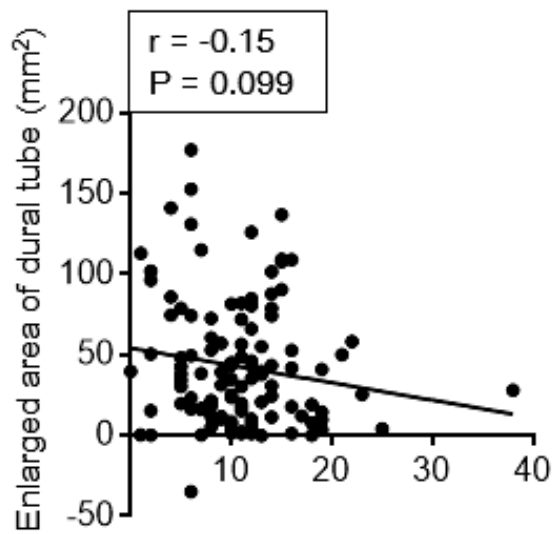

B

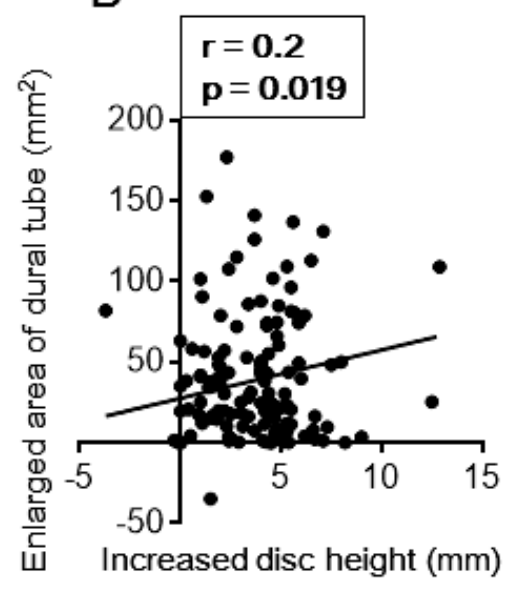

\section{Postoperative intervertebral angle $\left({ }^{\circ}\right)$}

Figure 2. The relationship between the postoperatively enlarged area of the dural tube and cage height $(\mathrm{mm})$, increased disc height $(\mathrm{mm})$, or postoperative intervertebral angle $\left(^{\circ}\right)$ were examined using Pearson's correlation coefficient. Enlargement of the area of dural tube was significantly correlated only with increased disc height.

plates, and expressed the value (a) divided by the full distance of the endplate (b) using a CT image. The area of dural tube was measured at the level of the disc using an axial MRI view with numerical computation software. Subsidence of the intervertebral cage was defined as settling of the cage by $1 \mathrm{~mm}$ or more. All radiology was performed by independent observers before and 6 months after surgery.

\section{Statistical analysis}

Analyses were conducted using Prism, version 4.0 (Graph Pad Software, La Jolla, CA). For all analyses, the level of significance was set at $p<0.05$.

\section{Results}

XLIF surgery associated with posterior stabilization, but without additional posterior spinal direct decompression, was performed on 53 indicated consecutive patients (38 women and 15 men; aged $69.2 \pm 10.2$ years) from July 2014 to September 2015. Lumbar degenerative disease re- quiring XLIF was lumbar spinal stenosis in 31 patients and adult spinal deformity in 22 patients. Levels treated by XLIF were L2-L3 in 30, L3-L4 in 42, and L4-L5 in 48 patients, respectively. The cage height used in XLIF surgery was $8 \mathrm{~mm}$ at 31 levels, $9 \mathrm{~mm}$ at 38 levels, $10 \mathrm{~mm}$ at 49 levels, and various others at 3 levels. All cages used were placed with $10^{\circ}$ of lordosis. No severe adverse events and no additional surgery were reported in the present study.

When comparing the preoperative period and 6 months post operatively, intervertebral disc height and angle were significantly increased at all levels (Fig. 1A). The area of the dural tube was significantly increased, whereas both the degree of disc bulging and thickness of flavum were significantly decreased at all disc levels (Fig. 1B). In addition, 10 patients showed postoperative subsidence of the disc cage. We examined the relationship among the postoperative enlarged area of the dural tube and cage height, increased disc height, and postoperative intervertebral angle. The enlargement of the area of the dural tube showed no correlation with cage height or postoperative intervertebral angle but 

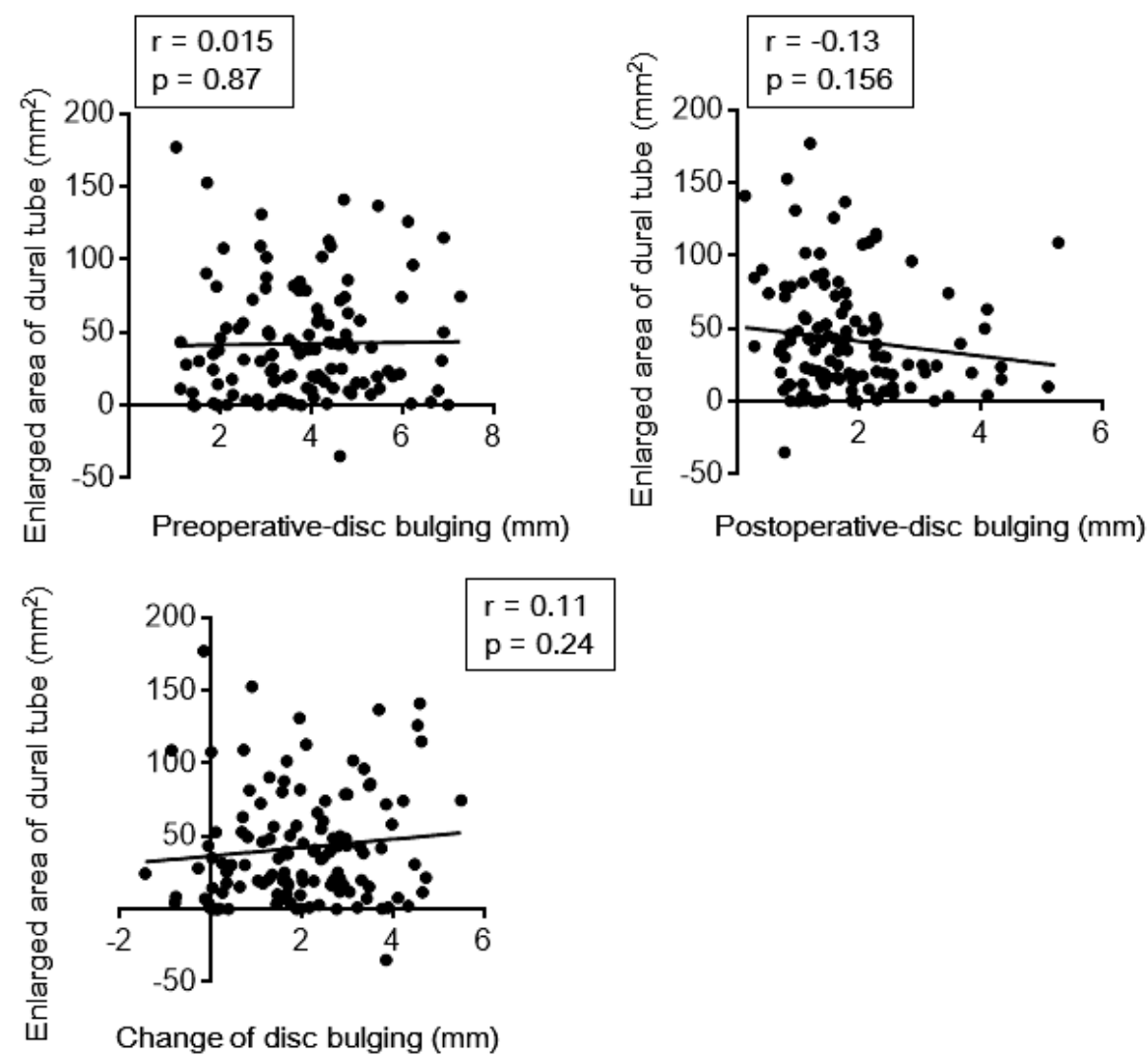

Figure 3. The relationship between the postoperatively enlarged area of the dural tube and pre- and postoperative disc bulging $(\mathrm{mm})$ and change of disc bulging $(\mathrm{mm})$ was examined using Pearson's correlation coefficient. Enlargement of the area of dural tube was not significantly correlated with any disc bulging.

was correlated with an increased disc height (Fig. 2A-C). We also examined the relationship among the postoperatively enlarged area of the dural tube and preoperative disc bulging, postoperative disc bulging, change of disc bulging after surgery, preoperative flavum thickness, postoperative flavum thickness, and change of flavum thickness after surgery. The enlargement of the area of the dural tube showed no correlation with preoperative disc bulging, postoperative disc bulging, or change of disc bulging (Fig. 3). However, the enlargement of the area of the dural tube showed significant correlation with preoperative flavum thickness and change of flavum thickness, but no correlation with postoperative flavum thickness (Fig. 4).

We examined the relationship between the enlarged area of the dural tube and cage placement at rostral and caudal endplates. The enlargement of the area of the dural tube showed a significant correlation with the cage placement at the rostral endplate, but no correlation with the cage placement at the caudal endplate (Fig. 5).

\section{Discussion}

Conventional surgical treatment includes direct decompression of nerve tissues in the presence or absence of arthrosis, whereas intraoperative techniques such as direct operation on nerves or dural tissues are potential reasons for postoperative complications ${ }^{3,4}$. Therefore, new indirect nerve decompression treatments such as XLIF and X-Stop (Medtronics, Minneapolis, MN) have been developed ${ }^{8,913)}$. Insertion of an X-Stop spacer into the region of the interspinous process prevents extension position and produces a local kyphosis at the level of symptoms ${ }^{14)}$ and may result in indirect decompression of nerve tissues at the level of lumbar spinal stenosis due to a reduction in the degree of flavum buckling ${ }^{13}$. A recent prospective randomized-controlled study demonstrated no significant differences in postoperative clinical outcomes between decompression surgery and the X-Stop operation 24 months after surgery ${ }^{14}$. By contrast, XLIF surgery uses a different mechanism for indirect decompression $^{10,11}$ and results in expanding an intervertebral disc space and a rigid support of the anterior region of spine with the broad surface of a cage. A previous study demonstrated an increase in average intervertebral disc height (41.9\%), foraminal height (13.5\%), foraminal area (24.7\%), and central canal diameter $(33.1 \%)$ at 43 lumbar levels ${ }^{12}$. The mechanism of indirect decompression by XLIF can be considered as a decrease in both the degree of buckling of hypertrophic degenerated flavum and protrusion of a degenerated disc into the spinal canal through an increase in disc height after the XLIF cage has been installed. The present study corroborated the idea of a decrease in flavum buckling as a more important consideration in obtaining indirect de- 
A

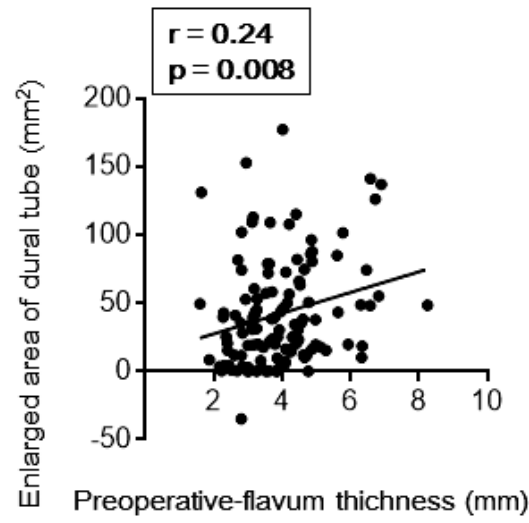

$\mathrm{C}$

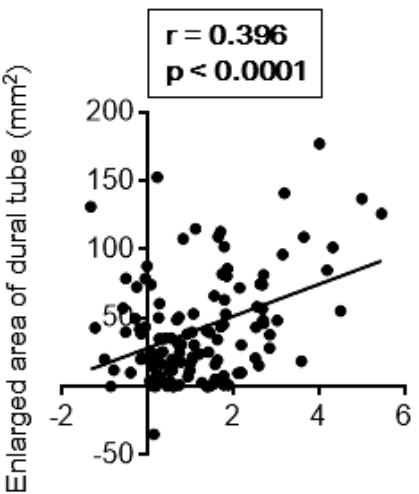

B

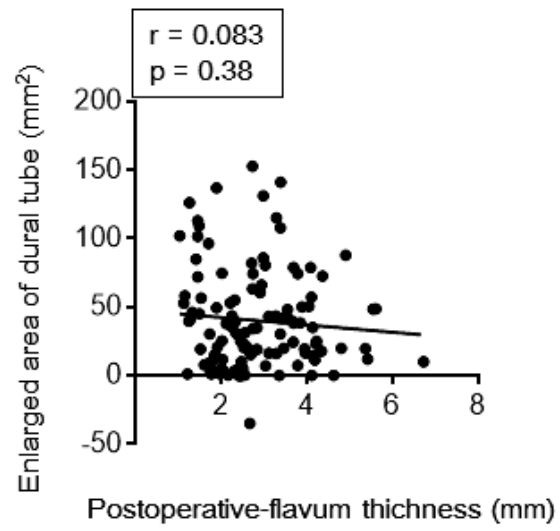

Change of flavum thichness $(\mathrm{mm})$

Figure 4. The relationship among the postoperatively enlarged area of the dural tube, preand postoperative flavum thickness $(\mathrm{mm})$, and change of disc bulging $(\mathrm{mm})$ was examined using Pearson's correlation coefficient. Enlargement of the area of the dural tube was significantly correlated with preoperative and change of flavum thickness.

compression than disc protrusion. A decrease or change in flavum thickness may be rapidly obtained after surgery, resulting in a quick improvement of neurological and other clinical outcomes. By contrast, enlargement of the area of the dural tube shows no correlation with preoperative disc bulging, postoperative disc bulging, or change of disc bulging. Decrease or change of disc bulging may only be obtained more than 6 months postoperatively in a slowly progressive manner.

Previous reports indicated additional decompression surgery was needed in 9\% (11 cases among 122 patients) and in $9.5 \%$ ( 2 cases among 21 patients $)^{11)}$. They listed spondylolisthesis from high grade facet arthropathy with instability, bony lateral recess stenosis, degenerative unstable spondylolisthesis, cage misplacement, locked facets such as correction of alignment blocked by osteophytes on the posterior surface of the articular processes and anterior osteophytes still impinging on the neural elements, congenital stenosis or congenitally short pedicles, uncontained disc herniation, calcified disc, osteophytes arising from the posterior endplates, synovial cysts, and symptoms unimproved with flexion as possible causes of inadequate outcome of indirect decompression with XLIF. In addition, subsidence of a cage leads to a loss of correction and may reduce the effect of indirect decompression ${ }^{15}$. Taller cage height, narrower cage width, and shorter cage length were reported to increase the risk of cage settling ${ }^{16}$. In our present series, the rate of cage subsidence was $17.5 \%$, which is almost the same rate as seen in previous studies ${ }^{15)}$.

We also examined whether the placement of a cage had a rostral or caudal endplate effect enlarging the spinal canal postoperatively and concluded that the postoperative area of the spinal canal was significantly correlated with the placement of the cage at the rostral endplate only, which suggested the importance of cage placement during surgery, particularly in the case of spondylolisthesis. However, a previous study examined the anterior and posterior border of the adjacent inferior vertebral body on midline images. Anterior cages were centered in the anterior $40 \%$ of the vertebral body $(21 \%)$, middle cages were centered in the middle $20 \%(64 \%)$, and posterior cages were centered in the posterior $40 \%$ (15\%) of 67 cages in 29 patients ${ }^{17)}$. The increase in foraminal area and the change in anterior disc height showed no significant change in terms of the cage being centered in the anterior, middle, or posterior aspect of the disc space. Another study reported XLIF cages were significantly more centrally located than cages for direct lumbar interbody fusion ${ }^{18)}$. The present study is limited as the cage placement evaluation was qualitative and it included only a relatively small number of cases. Nevertheless, we investi- 
A

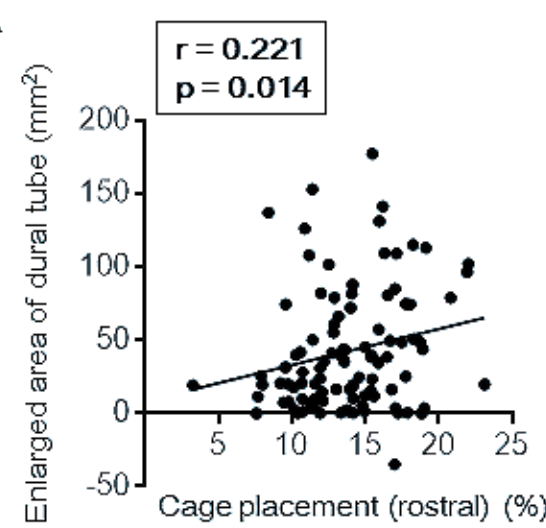

B

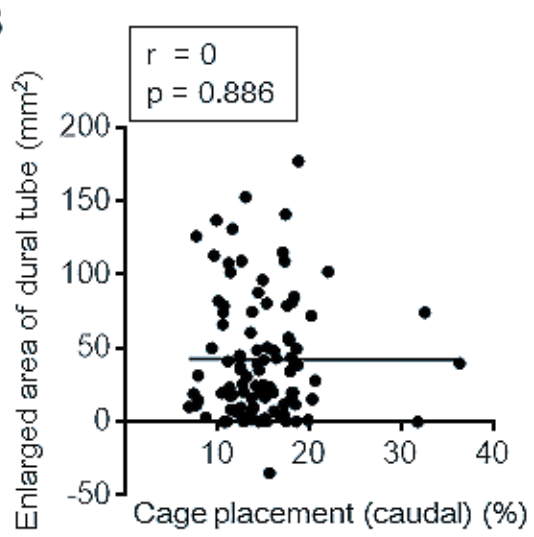

C

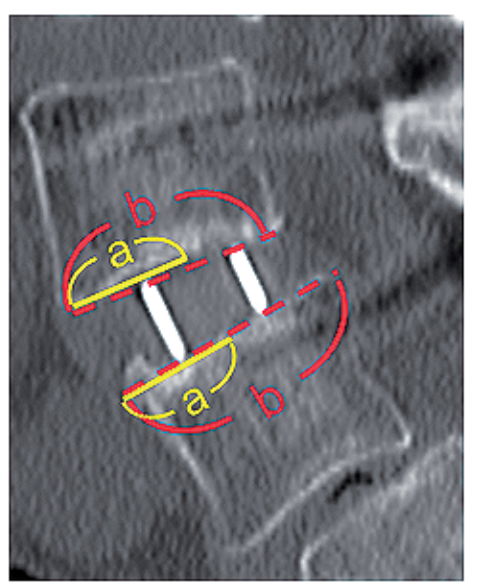

Figure 5. The distance (a) between the anterior edge and the center of the cage on both the rostral and caudal endplates was measured using a CT image and the value (a) divided by the full distance of the endplate (b) was expressed (c). The relationship between the postoperatively enlarged area of the dural tube and cage placement at the rostral and caudal endplates (\%) was examined using Pearson's correlation coefficient. Enlargement of the area of the dural tube was only significantly correlated with cage placement at the rostral endplate.

gated the cage placement more precisely by measuring the distances (a) between the anterior edge and center of cages on both rostral and caudal endplates and expressed the value (a) divided by the full distance of endplate (b) as shown in Fig. 5C using CT. Installing a cage in the middle of a disc space at the rostral endplate results in the rostral endplate becoming parallel to the caudal endplate and an increase in posterior disc height. This mechanism is effective for indirect decompression. When we installed a cage in the anterior of the disc space, we obtained a favorable correction of lordosis. We speculate that appropriate cage placement is important. Anterior placement is effective for correction of spinal alignment, whereas center placement is advantageous for indirect decompression.

The current study is limited by the relatively short postoperative observation period and radiological evaluation by only a single independent physician. Moreover, a precise relationship between radiological findings and clinical outcomes was not shown and we have not evaluated the differ- ence in cost-effectiveness between the XLIF surgery and decompression surgery.

\section{Conclusions}

The stabilizing effect of XLIF may contribute to favorable outcomes postoperatively. The present study demonstrated that a decrease in flavum buckling is a major consideration in obtaining indirect decompression. Center placement of an XLIF cage at the rostral endplate is advantageous for indirect decompression.

Conflicts of Interest: The authors declare that there are no conflicts of interest.

\section{References}

1. Daffner SD, Wang JC. The pathophysiology and nonsurgical treatment of lumbar spinal stenosis. Instr Course Lect. 2009; 58: 65768. 
2. Epstein NE. Surgical management of lumbar stenosis: decompression and indications for fusion. Neurosurg Focus. 1997; 3(2): e1.

3. Rompe JD, Eysel P, Zöllner J, et al. Degenerative lumbar spinal stenosis. Long-term results after undercutting decompression compared with decompressive laminectomy alone or with instrumented fusion. Neurosurg Rev. 1999; 22(2-3): 102-6.

4. Marquez-Lara A, Nandyala SV, Hassanzadeh H, et al. Sentinel events in lumbar spine surgery. Spine (Phila Pa 1976). 2014; 39 (11): 900-5

5. Lammli J, Whitaker MC, Moskowitz A, et al. Stand-alone anterior lumbar interbody fusion for degenerative disc disease of the lumbar spine: results with a 2-year follow-up. Spine (Phila Pa 1976) 2014; 39(15): E894-901.

6. Nourian AA, Cunningham CM, Bagheri A, et al. Effect of anatomic variability and level of approach on perioperative vascular complications with anterior lumbar interbody fusion. Spine (Phila Pa 1976). 2016; 41(2): E73-7.

7. Sasso RC, Best NM, Mummaneni PV, et al. Analysis of operative complications in a series of 471 anterior lumbar interbody fusion procedures. Spine (Phila Pa 1976). 2005; 30(6): 670-4.

8. Ozgur BM, Aryan HE, Pimenta L, et al. Extreme lateral interbody fusion (XLIF): a novel surgical technique for anterior lumbar interbody fusion. Spine J. 2006; 6(4): 435-43.

9. Billinghurst J, Akbarnia BA. Spine extreme lateral interbody fusion - XLIF. Curr Orthop Pract. 2009; 20(3): 238-51.

10. Malham GM, Parker RM, Goss B, et al. Indirect foraminal decompression is independent of metabolically active facet arthropathy in extreme lateral interbody fusion. Spine (Phila Pa 1976). 2014; 39 (22): E1303-10.

11. Malham GM, Parker RM, Goss B, et al. Clinical results and limitations of indirect decompression in spinal stenosis with laterally implanted interbody cages: results from a prospective cohort study.
Eur Spine J. 2015; 24 Suppl 3: 339-45.

12. Oliveira L, Marchi L, Coutinho E, et al. A radiographic assessment of the ability of the extreme lateral interbody fusion procedure to indirectly decompress the neural elements. Spine (Phila Pa 1976). 2010; 35(26 Suppl): S331-7.

13. Kuchta J, Sobottke R, Eysel P, et al. Two-year results of interspinous spacer (X-Stop) implantation in 175 patients with neurologic intermittent claudication due to lumbar spinal stenosis. Eur Spine J. 2009; 18(6): 823-9.

14. Strömqvist BH, Berg S, Gerdhem P, et al. X-stop versus decompressive surgery for lumbar neurogenic intermittent claudication: randomized controlled trial with 2-year follow-up. Spine (Phila Pa 1976). 2013; 38(17): 1436-42.

15. Le TV, Baaj AA, Dakwar E, et al. Subsidence of polyetheretherketone intervertebral cages in minimally invasive lateral retroperitoneal transpsoas lumbar interbody fusion. Spine (Phila Pa 1976). 2012; 37(14): 1268-73.

16. Tohmeh AG, Khorsand D, Watson B, et al. Radiographical and clinical evaluation of extreme lateral interbody fusion: effects of cage size and instrumentation type with a minimum of 1-year follow-up. Spine (Phila Pa 1976). 2014; 39(26): E1582-91.

17. Kepler CK, Sharma AK, Huang RC, et al. Indirect foraminal decompression after lateral transpsoas interbody fusion. J Neurosurg Spine. 2012; 16(4): 329-33.

18. Siu TL, Najafi E, Lin K. A radiographic analysis of cage positioning in lateral transpsoas lumbar interbody fusion. J Orthop. 2016; 14(1): $142-6$

Spine Surgery and Related Research is an Open Access article distributed under the Creative Commons Attribution - NonCommercial - NoDerivatives 4.0 International License. To view the details of this license, please visit (https://creativecommons.org/licenses/by - nc - nd/4.0/). 